OPEN ACCESS

Edited by:

Minming Zhang,

Zhejiang University, China

Reviewed by:

Falei Yuan,

Zhejiang Ocean University, China

Zhijun Zhang,

Shanghai Jiao Tong University, China

${ }^{*}$ Correspondence:

Guolin Ma

maguolin1007@qq.com

†These authors have contributed equally to this work

Specialty section:

This article was submitted to

Neurodegeneration,

a section of the journal

Frontiers in Neuroscience

Received: 18 September 2020 Accepted: 17 December 2020

Published: 21 January 2021

Citation:

Du L, Zhao Z, Liu X, Chen Y, Gao W Wang Y, Liu J, Liu B and Ma G (2021) Alterations of Iron Level in the Bilateral Basal Ganglia Region in Patients With Middle Cerebral Artery Occlusion.

Front. Neurosci. 14:608058.

doi: $10.3389 /$ fnins.2020.608058

\section{Alterations of Iron Level in the Bilateral Basal Ganglia Region in Patients With Middle Cerebral Artery Occlusion}

\author{
Lei $\mathrm{Du}^{1,2+}$, Zifang Zhao ${ }^{3+}$, Xiuxiu Liu ${ }^{1+}$, Yue Chen ${ }^{1}$, Wenwen Gao ${ }^{1}$, Yige Wang ${ }^{1}$, Jian Liu ${ }^{4}$, \\ Bing Liu ${ }^{1}$ and Guolin Ma ${ }^{1,2 *}$
}

${ }^{1}$ Department of Radiology, China-Japan Friendship Hospital, Beijing, China, ${ }^{2}$ Graduate School of Peking Union Medical College, Peking Union Medical College, Chinese Academy of Medical Sciences, Beijing, China, ${ }^{3}$ Department of Anesthesiology, Peking University First Hospital, Peking University, Beijing, China, ${ }^{4}$ Department of Ultrasound Diagnosis, China-Japan Friendship Hospital, Beijing, China

Background and Purpose: The purpose of this study was to explore the changes of iron level using quantitative susceptibility mapping (QSM) in the bilateral basal ganglia region in middle cerebral artery occlusion (MCAO) patients with long-term ischemia.

Methods: Twenty-seven healthy controls and nine patients with MCAO were recruited, and their QSM images were obtained. The bilateral caudate nucleus $(\mathrm{Cd})$, putamen $(\mathrm{Pt})$, and globus pallidus (Gp) were selected as the regions of interest (ROls). Susceptibility values of bilateral ROls were calculated and compared between the affected side and unaffected side in patients with MCAO and between patients with MCAO and healthy controls. In addition, receiver operating characteristic (ROC) curves were performed to evaluate the diagnostic capability of susceptibility values in differentiating healthy controls and patients with MCAO by the area under the curve (AUC).

Results: The susceptibility values of bilateral Cd were asymmetric in healthy controls; however, this asymmetry disappeared in patients with MCAO. In addition, compared with healthy controls, the average susceptibility values of the bilateral $\mathrm{Pt}$ in patients with MCAO were increased $(P<0.05)$, and the average susceptibility value of the bilateral Gp was decreased $(P<0.05)$. ROC curves showed that the susceptibility values of the Pt and $\mathrm{Gp}$ had a larger $\mathrm{AUC}$ ( $\mathrm{AUC}=0.700$ and 0.889, respectively).

Conclusion: As measured by QSM, the iron levels of the bilateral basal ganglia region were significantly changed in patients with MCAO. Iron dyshomeostasis in the basal ganglia region might be involved in the pathophysiological process of middle cerebral artery stenosis and occlusion. These findings may provide a novel insight to profoundly address the pathophysiological mechanisms of MCAO.

Keywords: middle cerebral artery occlusion, quantitative susceptibility mapping, iron, susceptibility, basal ganglia region 


\section{INTRODUCTION}

It is well-established that stroke has been one of the most common cerebrovascular diseases that could generate neuronal deaths and subsequent disability and mortality. According to pathological origins, stroke can be divided into two forms, ischemic and hemorrhagic stroke, and ischemic stroke accounts for the majority of patients (Mohan et al., 2009). Notably, middle cerebral artery stenosis and occlusion is the most frequent ischemic subtype in clinical practice and the most widely selected experimental model to elucidate the pathophysiology of cerebrovascular ischemic lesions. Although non-infectious neuroinflammation, oxidative stress, and metabolic disturbances of metal ions have been implicated in the process of middle cerebral artery occlusion (MCAO) (Fujioka et al., 2003; Kamel and Iadecola, 2012), the pathogenesis of MCAO has not been fully elucidated.

Iron, mainly stored in the form of ferritin or hemosiderin in brain tissue, is indispensable to the maintenance of normal brain function as it is an important cofactor for various enzymes involved in neurotransmitter synthesis, oxygen transport, electron transfer, and myelin generation (Connor et al., 2001; Munoz and Humeres, 2012). Regardless of the crucial roles in normal physiological functions, overloading of iron in the brain might trigger an abnormal release of toxic free radicals and consequent oxidative damage (Bolt and Marchan, 2010; Kell, 2010). Consistently, recent studies indicated that excessive iron deposition in brain tissue was closely linked to a variety of neurodegenerative diseases, such as Parkinson's disease (PD), Alzheimer's disease (AD), and stroke, which validated the underlying role of brain iron in the pathogenesis and progression of cognitive symptoms (Bishop et al., 2011; Acosta-Cabronero et al., 2013; Murakami et al., 2015; Du et al., 2016).

Compared with the traditional iron measurement techniques, susceptibility weighted imaging (SWI) (Schafer et al., 2009; Shmueli et al., 2009) and quantitative susceptibility mapping (QSM) can non-invasively and quantitatively detect the magnetic susceptibility values of brain tissue with higher specificity and sensitivity. Previous studies had found that the susceptibility value of tissue acquired by QSM was positively correlated with the iron level in deep gray matter nuclei (Langkammer et al., 2012; Deistung et al., 2013; Lim et al., 2013; Sun et al., 2015), which further verified the specific advantage in the measurements of tissue iron content. Currently, QSM has been increasingly used for the diagnosis, grading, and severity prediction of neurodegenerative diseases, both in clinical practice and experimental investigation.

The blood and oxygen in the basal ganglia regions are mostly supplied by the middle cerebral artery, and only a small part is supplied by the anterior cerebral artery and the choroid artery. Ischemia induced by MCAO could cause a variety of pathophysiological complications and subsequently result in brain function disorders and brain damage. Interestingly, the ischemic damages were found not only in the regions supplied by the MCA but also in deep regions such as the basal ganglia region, hippocampus, hypothalamus, and thalamus (Fujioka et al., 2003; El Amki et al., 2015; Pang et al., 2015). A possible explanation is the concomitant occlusions of deep and small cerebral arteries arising directly from the internal carotid artery, at the proximity of the origin of MCA (El Amki et al., 2015). Moreover, several studies have found that long-term ischemia could result in oxidative stress, non-infectious neuroinflammation, acidosis, and metabolic disturbances of metal ions such as calcium (Siesjo, 1988; Kamel and Iadecola, 2012; Yushmanov et al., 2013; Liu et al., 2014). However, it remains unknown whether the metabolism of iron in the basal ganglia regions would change after a long-term ischemia. We speculated that the iron level in the basal ganglia regions may change along with ischemic brain damage secondary to MCAO. The purpose of this study was to explore the changes of iron level using QSM in the bilateral basal ganglia region in MCAO patients with long-term ischemia.

\section{MATERIALS AND METHODS}

\section{Subjects}

This study protocol was approved by the Ethics Committee of China-Japan Friendship Hospital and informed consent of all the participants was obtained. The severity of stroke of all the participants was measured by the NIH Stroke Scale (NIHSS) scale. A total of 27 healthy controls and 9 adult patients with MCAO were enrolled in this research. The patients with MCAO visited the Department of Neurology of China-Japan Friendship Hospital between March 2018 and March 2019. The diagnosis of MCAO was made by neurological physicians based on clinical symptoms, CT results, conventional MRI, and MR angiography. Patients with MCAO were selected if they met the following criteria: (a) the MR image has no artifacts; (b) the clinical information of the participants is complete and the participants had no other brain diseases, such as tumors or dementia; and (c) the participants had no visual and hearing impairment, capable of coordinating with the completion of scale and MRI examination. There were five cases of left middle cerebral artery occlusion and four cases of right middle cerebral artery occlusion in the patient group. There were five males and four females, with an average age of $61.7 \pm 12.3$ years $(38-79$ years). All patients had chronic ischemia and were right-handed. Table 1 shows the detailed clinical information of the nine patients with MCAO.

Healthy controls were recruited from the local communities. Inclusion criteria were as follows: (a) ages range 54-79 (including 54 and 7 years); (b) being right-handed; (c) the patient has no basic diseases such as hypertension and diabetes, no cognitive impairment, and no family history of neurological and psychiatric illness; and (d) MRI examination reveals only small lacunar infarcts. Healthy controls suffering from cardiovascular, neurologic, metabolic, and psychiatric disorders or brain abnormalities were excluded from this study. Finally, 27 healthy controls were enrolled in the present study, 9 males and 18 females, with an average age of $65.1 \pm 7.6$ years ( $54-79$ years). Table 2 shows all the subjects' clinical information. All subjects received T1-weighted imaging, T2-weighted imaging, 3D T1weighted imaging, and quantitative susceptibility mapping. 
TABLE 1 | Detailed clinical information of nine patients with MCAO.

\begin{tabular}{|c|c|c|c|c|c|c|c|c|c|}
\hline Patient & Sex & Age & Affected side & Smoke & Drink & Hypertension & Diabetes & Handedness & Duration (years) \\
\hline 1 & $\mathrm{M}$ & 38 & Right & Y & $\mathrm{N}$ & Y & $\mathrm{N}$ & Right & 2 \\
\hline 2 & $\mathrm{M}$ & 61 & Left & $N$ & Y & $\mathrm{N}$ & $\mathrm{N}$ & Right & 5 \\
\hline 3 & $\mathrm{~F}$ & 57 & Left & $\mathrm{N}$ & $\mathrm{N}$ & Y & $\mathrm{N}$ & Right & 6 \\
\hline 4 & $\mathrm{~F}$ & 64 & Right & $\mathrm{N}$ & $\mathrm{N}$ & Y & $\mathrm{N}$ & Right & 2 \\
\hline 5 & $\mathrm{M}$ & 50 & Left & Y & Y & Y & $\mathrm{N}$ & Right & 1 \\
\hline 6 & $\mathrm{~F}$ & 79 & Left & $\mathrm{N}$ & $\mathrm{N}$ & $\mathrm{N}$ & $N$ & Right & 7 \\
\hline 7 & $\mathrm{~F}$ & 71 & Right & $\mathrm{N}$ & $\mathrm{N}$ & Y & $N$ & Right & 3 \\
\hline 8 & $\mathrm{M}$ & 63 & Left & $\mathrm{N}$ & $\mathrm{N}$ & Y & $\mathrm{N}$ & Right & 4 \\
\hline 9 & $\mathrm{M}$ & 72 & Right & $\mathrm{N}$ & $\mathrm{N}$ & Y & Y & Right & 6 \\
\hline
\end{tabular}

MCAO, middle cerebral artery occlusion; $M$, male; $F$, female; $Y$, yes; $N$, no.

TABLE 2 | Demographic data of healthy controls and patients with MCAO.

\begin{tabular}{lccc}
\hline & Healthy & MCAO & $P$-values \\
\hline No. & 27 & 9 & - \\
Sex (male/female) & $9 / 18$ & $5 / 4$ & $P>0.05$ \\
Age (years) & $65.1 \pm 7.6(54-79)$ & $61.7 \pm 12.3(38-79)$ & $P>0.05$ \\
Educations & $10.4 \pm 3.8$ & - & - \\
Duration (years) & - & $4.0 \pm 2.1(1-7)$ & - \\
Handedness (right) & 27 & 9 & $P>0.05$
\end{tabular}

-, not significant or not measured; MCAO, middle cerebral artery occlusion.

\section{MR Imaging Protocol}

A 3.0-T MRI scanner (GE Healthcare, Discovery MR750, Milwaukee, USA) equipped with an eight-channel head coil was used to acquire the data. All participants underwent a T2-weighted imaging scan to exclude craniocerebral organic diseases.

A 3D gradient-echo (GRE) sequence was used for quantitative susceptibility mapping. The parameters were field of view (FOV) $=256 \times 256 \mathrm{~mm}$, matrix size $=256 \times 256$, slice thickness $=$ $1.0 \mathrm{~mm}$, echo time $(\mathrm{TE})=3.2 \mathrm{~ms}$, repetition time $(\mathrm{TR})=22.9 \mathrm{~ms}$, flip angle $(\mathrm{FA})=12^{\circ}$, and scan time $=4 \mathrm{~min} 24 \mathrm{~s}$.

A 3D T1WI structure image was reconstructed using threedimensional fast spoiled gradient-echo sequences (3D FSPGR): FOV $=256 \times 256 \mathrm{~mm}$, matrix size $=256 \times 256$, slice thickness $=1.0 \mathrm{~mm}$, TR $=6.7 \mathrm{~ms}$, $\mathrm{TE}=\min$ full, $\mathrm{FA}=12^{\circ}$, number of slices $=192$, and scan time $=4 \mathrm{~min} 10 \mathrm{~s}$.

\section{QSM}

We performed several processing steps to calculate the quantitative susceptibility mapping from the obtained MR phase images. Firstly, a Laplacian-based phase unwrapping was used to perform the phase unwrapping images. Then, we acquired a brain mask using skull-stripping the GRE magnitude image obtained at a TE of $10 \mathrm{~ms}$. Secondly, the phase unwrapping images were divided by $2 \pi^{*} \mathrm{TE}$ to get images of the frequency shift in hertz for each echo. Thirdly, background fields were removed with the variable spheric kernel size sophisticated harmonic artifact decrease for the phase-data (V-SHARP) method with a regularization parameter of 0.05 and a maximum radius of $4 \mathrm{~mm}$. Compared with single-echo reconstruction, we averaged the resulting images of all five echoes to acquire a better signal-tonoise ratio after elimination of background fields. Finally, inverse dipole calculations using a least squares and QR factorizationbased minimization was performed to get the QSM imaging.

\section{Image Segmentation}

In the present study, the caudate nucleus $(\mathrm{Cd})$, putamen $(\mathrm{Pt})$, and globus pallidus ( $\mathrm{Gp}$ ) were selected as the regions of interest (ROIs) (Figure 1A). Firstly, the GRE quantitative susceptibility mapping was co-registered to the $3 \mathrm{D}$ T1WI, which is to better define the boundaries of each gray nucleus. The ROIs were then manually segmented by two radiologists (Lei Du, 5 years of working experience; Yue Chen, 2 years of working experience) slice by slice using the FuncTool on a GE AW4.6 workstation (GE Healthcare, Milwaukee, WI). They were blinded to clinical and MR imaging information. The susceptibility value and size of three successive slices were acquired in each ROI to eliminate the effects of the occasional case. Also, the average susceptibility values of each ROI were calculated. The unit of susceptibility value was parts per million (ppm).

\section{Statistical Analysis}

Between the patient group and the healthy control group, age was compared using two-sample $t$-test, and gender and handedness were compared using the chi-square $\left(\chi^{2}\right)$ test. The data of age and education was shown in the form of mean \pm SD.

The interobserver agreement of two observers was assessed using the intraclass correlation coefficient (ICC), and ICC $<0.40$ is poor, $0.40-0.59$ is fair, $0.60-0.74$ is good, and $>0.74$ is excellent (Oppo et al., 1998).

The paired $t$-test was used to assess the differences of susceptibility values between the left and right hemispheres in healthy controls and between the affected side and the unaffected side in patients with MCAO. Comparisons between patients with MCAO and the healthy controls were investigated using twosample $t$-test. Moreover, receiver operating characteristic (ROC) curves were performed to evaluate the diagnostic capability of susceptibility values in differentiating patients with MCAO and 
A

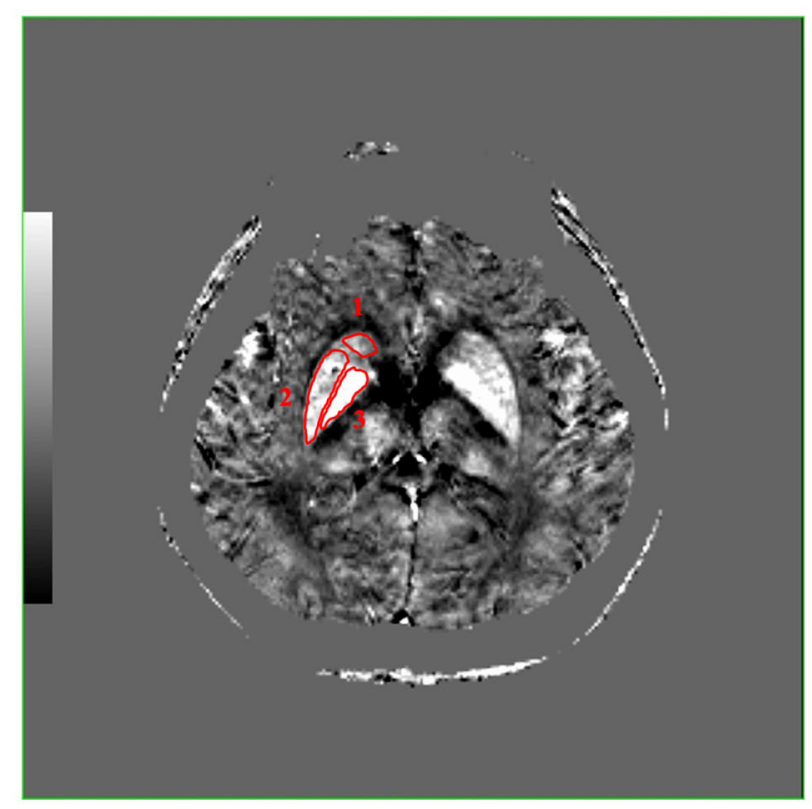

B

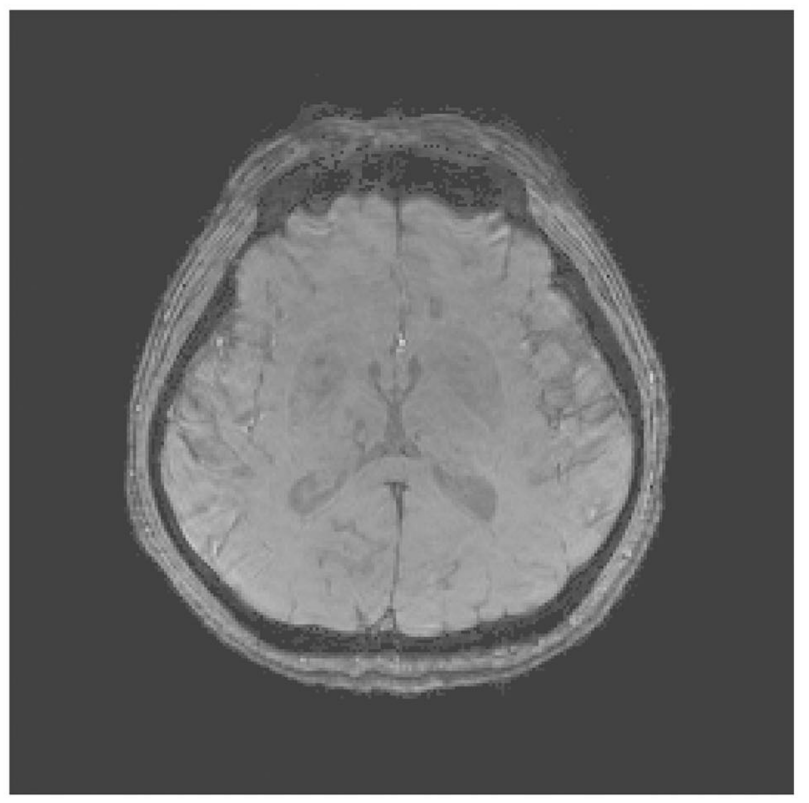

FIGURE 1 | These are QSM (A) and SWI (B) images of a 50-year-old male patient with MCAO at the same level, and the caudate nucleus (Cd), putamen (Pt), and globus pallidus (Gp) (number 1-3, separately) were selected as regions of interest (ROIs), which were encircled in red line in QSM image. Compared with SWI image, QSM image can show the boundary of the Cd, Pt, and Gp more clearly. QSM, quantitative susceptibility mapping; SWI, susceptibility weighted imaging; MCAO, middle cerebral artery occlusion; ROls, regions of interest.

healthy controls by the area under the curve (AUC) $P<0.05$ was considered to be statistically significant.

\section{RESULTS}

\section{Characteristics of All the Participants}

The demographic information of all the participants is summarized in Table 2. Finally, 9 MCAO patients ( 5 males and 4 females, mean age $61.7 \pm 12.3$ years, range 38-79 years) and 27 healthy controls ( 9 males and 18 females, mean age $65.1 \pm$ 7.6 years, range $54-79$ years, education $10.4 \pm 3.8$ years) were enrolled in this study. All participants are right-handed. There were five cases of left middle cerebral artery occlusion and four cases of right middle cerebral artery occlusion in the patient group. Then, the left and right gray nuclei in each ROI were divided into the affected side group and the unaffected side group in patients with MCAO. From Table 2, we knew that gender, age, and handedness between the patient group and healthy control group are matched $(P>0.05)$. The interobserver variability was assessed by ICC, and a good agreement was acquired between two radiologists for the susceptibility values (Table 3 ).

The QSM image showed the location of the bilateral Cd, Pt, and $\mathrm{Gp}$ in a 50-year-old male patient with MCAO (Figure 1A). Figure 1A shows that there were significant differences between the ROI and surrounding brain regions as seen by the naked eyes, indicating that the iron level in the $\mathrm{Cd}, \mathrm{Pt}$, and $\mathrm{Gp}$ is more than that in the surrounding brain region. Figure 1B displays a SWI image at the same level as QSM. Compared with the SWI image,
TABLE 3 | Intraclass correlation coefficient (ICC) of bilateral caudate nucleus (Cd), putamen (Pt), and globus pallidus (Gp) in patients with MCAO and healthy controls.

\begin{tabular}{llcc}
\hline ROIs & $\begin{array}{c}\text { ICC of the left } \\
\text { hemisphere }(\mathbf{9 5 \%} \mathbf{~ C l})\end{array}$ & $\begin{array}{c}\text { ICC of the right } \\
\text { hemisphere }(\mathbf{9 5 \%} \mathbf{~ C l})\end{array}$ \\
\hline MCAO & Cd & $0.973(0.881$ to 0.994$)$ & $0.448(-1.446$ to 0.876$)$ \\
& Pt & $0.987(0.943$ to 0.997$)$ & $0.313(-2.047$ to 0.845$)$ \\
& Gp & $0.877(0.455$ to 0.972$)$ & $0.916(0.628$ to 0.981$)$ \\
Healthy & Cd & $0.259(-1.309$ to 0.762$)$ & $0.742(0.196$ to 0.917$)$ \\
& Pt & $0.706(0.085$ to 0.906$)$ & $0.555(-0.388$ to 0.857$)$ \\
& Gp & $0.776(0.303$ to 0.928$)$ & $0.796(0.364$ to 0.934$)$ \\
\hline
\end{tabular}

ICC, intraclass correlation coefficient; Cd, caudate nucleus; Pt, putamen; Gp, globus pallidus; MCAO, middle cerebral artery occlusion; ROls, regions of interest; $\mathrm{Cl}$, confidence interval.

the QSM image could show the boundary of the Cd, Pt, and Gp more clearly.

\section{Comparisons of Susceptibility Values Between the Affected Side and Unaffected Side in Patients With MCAO and Between Left and Right in Healthy Controls}

The mean and SD of the susceptibility values of the Cd, Pt, and $\mathrm{Gp}$ in all participants are summarized in Tables 4, 5. 
patients and healthy controls, except the $\mathrm{Cd}$ of the patient group. There was no significant difference between the healthy group and the MCAO patient group both in the affected side and unaffected side of the $\mathrm{Cd}$. Figure 3 shows the comparisons between the healthy group and the patient group in the Pt and $\mathrm{Gp}$. Figure 3 shows that the susceptibility values of the Pt were significantly higher in patients with MCAO than those in the controls $(P<0.05)$, and the susceptibility values of the Gp were significantly lower in patients with MCAO than those in the controls $(P<0.05)$.

The susceptibility values of the bilateral Pt in MCAO patients were significantly higher than those in the controls. Figure 4 shows that the AUC of average susceptibility values of the bilateral Gp (AUC $=0.889$ ) was larger than the others, including the left $\mathrm{Cd}$, right $\mathrm{Cd}$, and bilateral Pt $(\mathrm{AUC}=0.761,0.679$, and 0.700 , respectively). The cutoff of the susceptibility value of $\mathrm{Pt}$ was 0.0996 . The sensitivity and specificity for identifying patients with MCAO from the controls were 66.7 and $66.7 \%$, respectively. In contrast, the susceptibility values of the bilateral $\mathrm{Gp}$ in patients with MCAO were significantly lower than those in the controls $(P$ $<0.05$ ). The cutoff of the susceptibility value for Gp was 0.1408 . The sensitivity and specificity for differentiating patients with MCAO from the controls were 77.8 and $81.5 \%$.

\section{DISCUSSION}

In the current study, susceptibility values acquired by QSM were used to investigate the alterations of the iron level in the bilateral basal ganglia region in patients with MCAO. We found some alterations of susceptibility values in patients with MCAO and some significant differences between patients with MCAO and healthy controls. More specifically, the susceptibility values of the $\mathrm{Pt}$ in patients with MCAO were significantly higher than those in healthy controls, and the susceptibility values of the bilateral Gp were significantly lower in patients with MCAO than in the healthy controls. In addition, the results also showed that the susceptibility values of bilateral $\mathrm{Cd}$ were asymmetric in healthy controls; however, the asymmetry disappeared in patients with MCAO. There were no significant differences between the affected side and the unaffected side in patients with MCAO.

The human cerebrovascular system has several compensatory neuroprotective approaches in response to ischemic attack, which help to diminish the neuronal damage. During the disease process of middle cerebral artery stenosis or occlusion, the compensatory neuroprotective mechanisms would be automatically activated if ipsilateral cerebral blood flow is insufficient and impacts normal brain function. Principal compensation approaches include the opening of communicating branches of Willis circle, the increase of vascular internal diameter, bloodstream speeding, and so on. The above compensatory reflections may contribute to the abnormal iron metabolism and consequent alterations of iron level in the bilateral basal ganglia region, which was found in the present study. However, further research is necessary to investigate it.

Iron plays a vital role in maintaining numerous fundamental biological functions of the human brain. However, disorders of iron homeostasis or excessive iron accumulation may lead to abnormal generation of toxic free radicals and consequent oxidative damage (Bolt and Marchan, 2010; Kell, 2010), which are molecular and pathological characteristics of some neurodegenerative diseases such as Alzheimer's disease, Parkinson's disease, and Huntington's disease (Ward et al., 2014; Masaldan et al., 2019; Thomas et al., 2020).

In the current study, we found that a significant increase of iron levels in the Pt was presented in patients with MCAO,
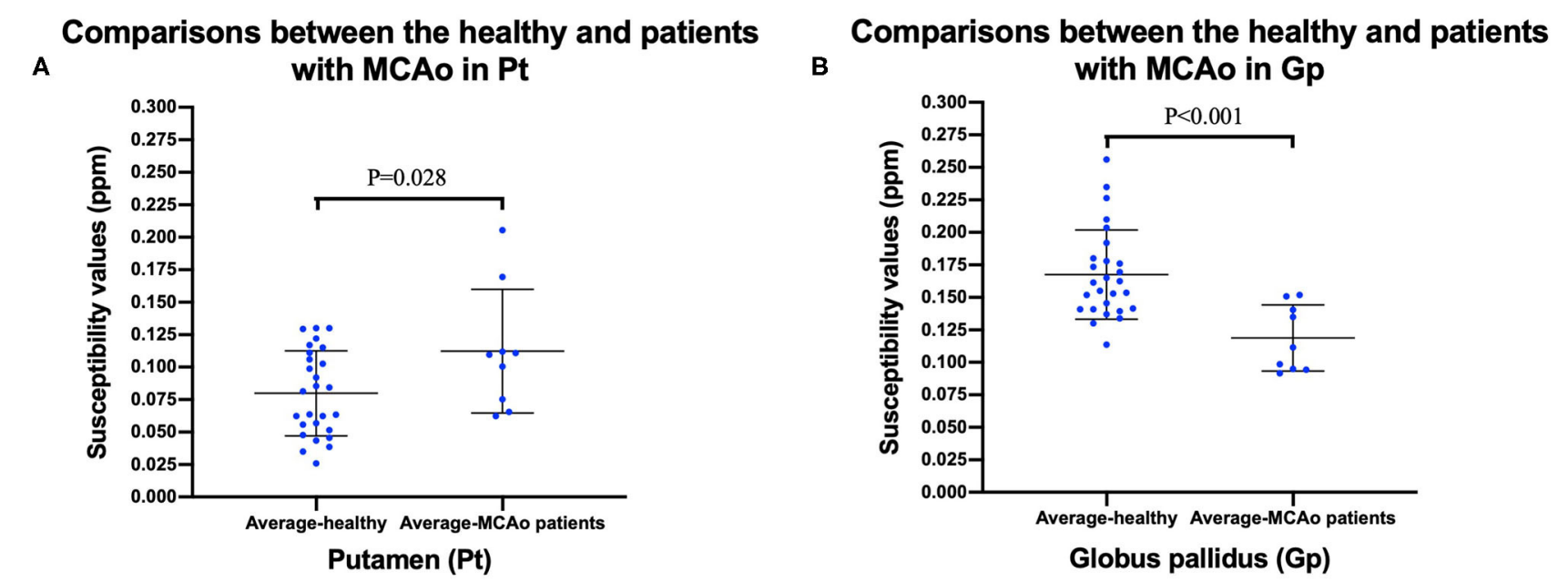

FIGURE 3 | Comparisons between healthy controls and patients with MCAO in putamen (Pt) (A), and globus pallidus (Gp) (B). Data was mean $\pm \mathrm{SD}, \mathrm{N}=27$ for healthy controls, and $N=9$ for patients with MCAO. Two sample $t$-test was conducted between two groups and $P<0.05$ was considered as significant. MCAO, middle cerebral artery occlusion; Cd, caudate nucleus; Pt, putamen; Gp, globus pallidus; SD, standard deviation. 


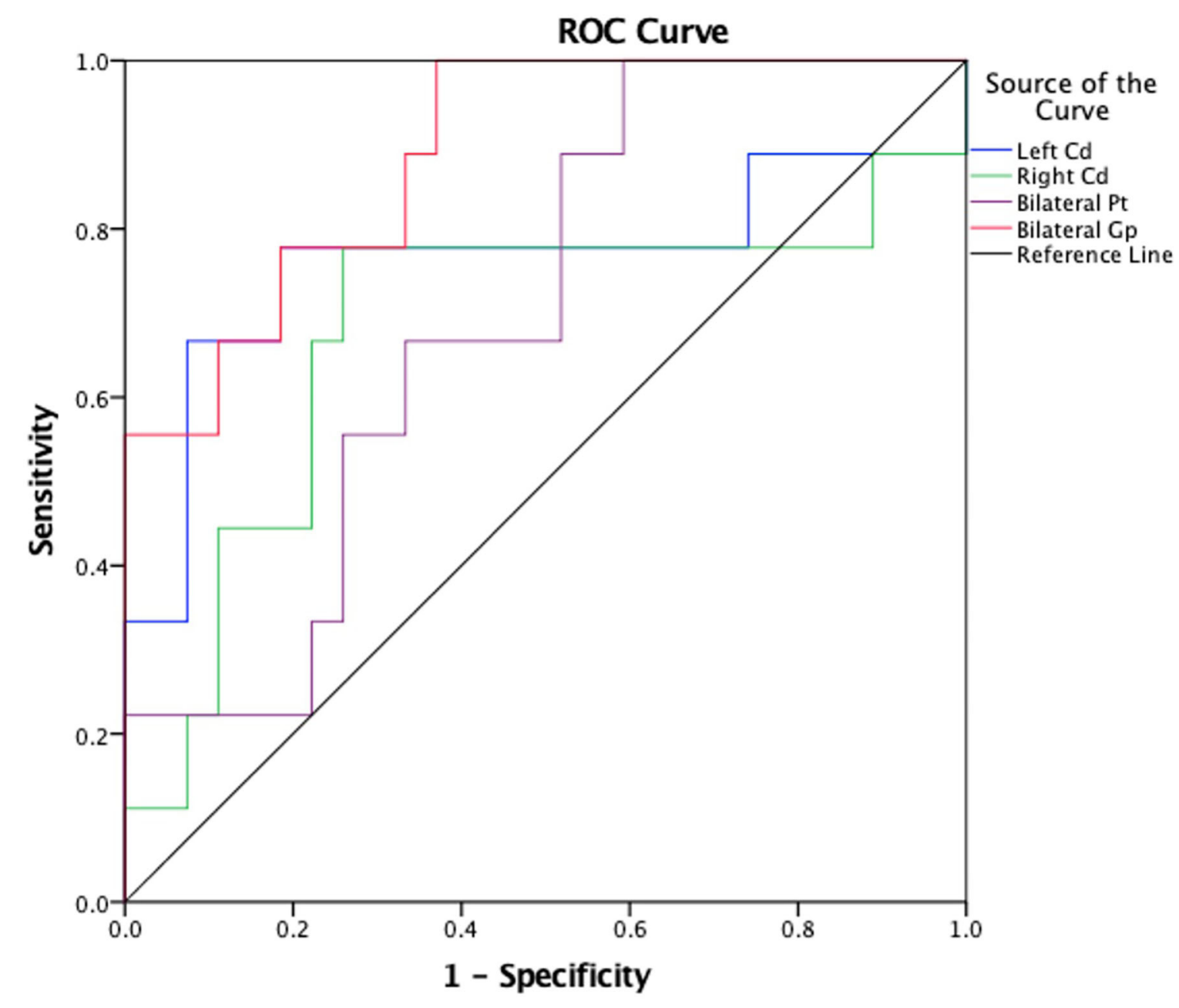

FIGURE 4 | Receiver operating characteristic (ROC) curve was generated using the left Cd, right Cd, bilateral Pt, bilateral Gp for differentiating MCAO patients and healthy controls. This figure showed that the area under the curve (AUC) of average susceptibility values of the Gp (AUC $=0.889$ ) was larger than others, including left

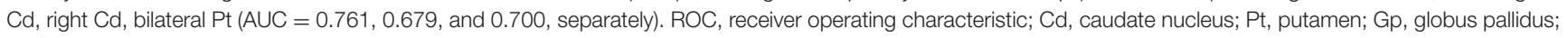
$\mathrm{MCAO}$, middle cerebral artery occlusion; AUC, area under the curve.

indicating that MCAO could result in pathological changes of microscopic and molecular properties in the basal ganglia region. Possible interpretations for this pathological alteration are as follows: Firstly, inadequate blood flow and oxygen supply induced by MCAO can result in the proliferation, activation, and migration of astrocytes and microglia ( $\mathrm{Hu}$ et al., 2015; Gulke et al., 2018; Zhang et al., 2019). Astrocytes and microglia significantly impact iron transport or storage mechanisms, which were involved in iron-mediated toxicity (Bishop et al., 2011). Secondly, ischemic stroke, including MCAO, can trigger a cascading pathological noninfectious neuroinflammation and the subsequent excessive release of proinflammatory cytokines, including interleukin-1 (IL-1), tumor necrosis factor alpha (TNF$\alpha$ ), and interleukin-6 (IL-6) (Fujioka et al., 2003; Wang et al., 2007; Chamorro et al., 2016). Neuroinflammation might affect the iron homeostasis in the CNS glia. Abnormal TNF- $\alpha$ release could result in increased uptake and retention of iron both in astrocytes and microglia (Rathore et al., 2012). Thirdly, we speculated that injury of the blood-brain barrier induced by ischemia attack might be another reason leading to iron deposition. It is well-recognized that the blood-brain barrier plays a crucial role in regulating the physiological transportation and metabolism of various endogenous substances and ions, including iron (Khan et al., 2018; Chiou et al., 2019; Jackson et al., 2019; Linville et al., 2019). Ischemia-related damage of endothelial cells of the blood-brain barrier significantly impairs the normal absorption of iron, and excessive amounts of iron consequently cross into the brain tissue and can be absorbed by microglia and astrocytes (McCarthy and Kosman, 2015; Simpson et al., 2015; Bu et al., 2019; Yan and Zhang, 2019), which eventually leads to increased iron level in the Pt. 
Accumulating studies indicated that iron overloading and the consequent spontaneous release of neurotoxic free iron in these brain regions could result in neuronal death and memory dysfunction (Rouault, 2001). It had been demonstrated that neuronal death of the $\mathrm{Cd}$ and $\mathrm{Pt}$ could result in a series of neurocognitive dysfunctions including cognition deficit (Petty et al., 1996; Du et al., 2018). Our findings in this study provided a probable explanation regarding the neurological symptoms manifested in MCAO patients.

Interestingly, the iron level in the bilateral Gp in MCAO patients was significantly decreased when compared with that in healthy controls. Possible explanations are as follows: Firstly, a significant fluctuation of brain iron may occur during the poststroke recovery. Following the ischemic attack, various endogenous brain repair processes would be activated and engaged in functional recovery (Zhang and Chopp, 2015; Zhao and Willing, 2018). Notably, it had been demonstrated that iron metabolism was involved in the spontaneous repair in chronic ischemic brain regions (Shin et al., 2018; Kim et al., 2020). We postulated that the active iron transport and the imbalance of iron metabolism along with the brain repair process may result in a decreased iron level in some brain nucleus. Secondly, the TNF- $\alpha$ and interferon- $\gamma$ expressed by activated microglia presented an iron-mediated toxic effect on oligodendrocytes (Zhang et al., 2005), which could trigger a subsequent release of intracellular iron (Zhang et al., 2006). The liberated iron could be detoxified and cleared from the brain region, which ultimately decreased the iron level. In addition, the decrease of blood supply and iron-rich erythrocytes after MCAO might be another reason for this phenomenon. Decreased cerebral blood flow implied a significant reduction of iron coming from erythrocytes in affected brain regions, such as the Gp.

The limitations of this study should not be neglected. First, the number of patients with MCAO enrolled in this study was limited, and the conclusions need to be verified by future studies with a larger sample size. Second, the voxel of imaging was large, and a single voxel displayed an average measurement of the neuronal environment. Third, it is hard to completely avoid the slight errors when drawing the ROIs due to the undefined nucleus boundary in current images. Therefore, further study should be conducted to thoroughly explore the potential pathophysiological roles of the basal ganglia region in patients with MCAO and the correlations between the occurrence and progression of ischemia and the alterations of the iron level in the basal ganglia region.

\section{REFERENCES}

Acosta-Cabronero, J., Williams, G. B., Cardenas-Blanco, A., Arnold, R. J., Lupson, V., and Nestor, P. J. (2013). In vivo quantitative susceptibility mapping (QSM) in Alzheimer's disease. PLoS ONE 8:e81093. doi: 10.1371/journal.pone. 0081093

Bishop, G. M., Dang, T. N., Dringen, R., and Robinson, S. R. (2011). Accumulation of non-transferrin-bound iron by neurons, astrocytes, and microglia. Neurotox. Res. 19, 443-451. doi: 10.1007/s12640-010-9195-x

\section{CONCLUSIONS}

As measured by QSM, iron levels of the bilateral basal ganglia region were significantly changed in patients with MCAO. Iron dyshomeostasis in the basal ganglia region might be involved in the pathophysiological process of middle cerebral artery stenosis and occlusion. These findings may provide a novel insight to profoundly address the pathophysiological mechanisms of MCAO.

\section{DATA AVAILABILITY STATEMENT}

The original contributions presented in the study are included in the article/supplementary materials, further inquiries can be directed to the corresponding author.

\section{ETHICS STATEMENT}

The studies involving human participants were reviewed and approved by China-Japan Friendship Hospital. The participants provided their written informed consent to participate in this study. Written informed consent was obtained from the individuals for the publication of any potentially identifiable images or data included in this article.

\section{AUTHOR CONTRIBUTIONS}

$\mathrm{LD}, \mathrm{ZZ}$, and XL designed this research, analyzed the MRI data, and drafted this manuscript. LD and YC drew the regions of interest. WG, YW, JL, and BL did the MRI scanning. GM revised the whole manuscript. All authors contributed to the article and approved the submitted version.

\section{FUNDING}

This study was supported by the National Key Research and Development Program of China (Grant Nos. 2020YFC2003903, 2019YFC0120903, and 2016YFC1307001) and by grants from the National Natural Science Foundation of China (NSFC) (Grant Nos. 81971585, 81571641, and 91959123).

\section{ACKNOWLEDGMENTS}

We thank all the patients and healthy controls who participated in our study. The authors thank Dr. Lizhi Xie from GE Healthcare for helping in solving MR technical problems.

Bolt, H. M., and Marchan, R. (2010). Iron dysregulation: an important aspect in toxicology. Arch. Toxicol. 84, 823-824. doi: 10.1007/s00204010-0610-0

Bu, X. L., Xiang, Y., and Guo, Y. (2019). The role of iron in amyotrophic lateral sclerosis. Adv Exp Med Biol. 1173, 145-152. doi: 10.1007/978-981-13-9589-5_8

Chamorro, A., Dirnagl, U., Urra, X., and Planas, A. M. (2016). Neuroprotection in acute stroke: targeting excitotoxicity, oxidative and nitrosative stress, and inflammation. Lancet Neurol. 15, 869-881. doi: 10.1016/S1474-4422(16)00114-9 
Chiou, B., Neal, E. H., Bowman, A. B., Lippmann, E. S., Simpson, I. A., and Connor, J. R. (2019). Endothelial cells are critical regulators of iron transport in a model of the human blood-brain barrier. J. Cereb. Blood Flow Metab. 39, 2117-2131. doi: $10.1177 / 0271678 \times 18783372$

Connor, J. R., Menzies, S. L., Burdo, J. R., and Boyer, P. J. (2001). Iron and iron management proteins in neurobiology. Pediatr. Neurol. 25, 118-129. doi: 10.1016/S0887-8994(01)00303-4

Deistung, A., Schafer, A., Schweser, F., Biedermann, U., Turner, R., and Reichenbach, J. R. (2013). Toward in vivo histology: a comparison of quantitative susceptibility mapping (QSM) with magnitude-, phase-, and R2*-imaging at ultra-high magnetic field strength. Neuroimage 65, 299-314. doi: 10.1016/j.neuroimage.2012.09.055

Du, G., Liu, T., Lewis, M. M., Kong, L., Wang, Y., Connor, J., et al. (2016). Quantitative susceptibility mapping of the midbrain in Parkinson's disease. Mov. Disord. 31, 317-324. doi: 10.1002/mds.26417

Du, L., Zhao, Z., Cui, A., Zhu, Y., Zhang, L., Liu, J., et al. (2018). Increased iron deposition on brain quantitative susceptibility mapping correlates with decreased cognitive function in Alzheimer's disease. ACS Chem. Neurosci. 9, 1849-1857. doi: 10.1021/acschemneuro.8b00194

El Amki, M., Clavier, T., Perzo, N., Bernard, R., Guichet, P. O., and Castel, H. (2015). Hypothalamic, thalamic and hippocampal lesions in the mouse MCAO model: potential involvement of deep cerebral arteries? J. Neurosci. Methods 254, 80-85. doi: 10.1016/j.jneumeth.2015.07.008

Fujioka, M., Taoka, T., Matsuo, Y., Mishima, K., Ogoshi, K., Kondo, Y., et al. (2003). Magnetic resonance imaging shows delayed ischemic striatal neurodegeneration. Ann Neurol. 54, 732-747. doi: 10.1002/ana.10751

Gulke, E., Gelderblom, M., and Magnus, T. (2018). Danger signals in stroke and their role on microglia activation after ischemia. Ther. Adv. Neurol. Disord. 11:1756286418774254. doi: 10.1177/1756286418774254

Hu, X., Leak, R. K., Shi, Y., Suenaga, J., Gao, Y., Zheng, P., et al. (2015). Microglial and macrophage polarization-new prospects for brain repair. Nat. Rev. Neurol. 11, 56-64. doi: 10.1038/nrneurol.2014.207

Jackson, S., Meeks, C., Vezina, A., Robey, R. W., Tanner, K., and Gottesman, M. M. (2019). Model systems for studying the blood-brain barrier: applications and challenges. Biomaterials 214:119217. doi: 10.1016/j.biomaterials. 2019.05.028

Kamel, H., and Iadecola, C. (2012). Brain-immune interactions and ischemic stroke: clinical implications. Arch. Neurol. 69, 576-581. doi: $10.1001 /$ archneurol.2011.3590

Kell, D. B. (2010). Towards a unifying, systems biology understanding of largescale cellular death and destruction caused by poorly liganded iron: parkinson's, huntington's, alzheimer's, prions, bactericides, chemical toxicology and others as examples. Arch. Toxicol. 84, 825-889. doi: 10.1007/s00204-010-0577-x

Khan, A. I., Liu, J., and Dutta, P. (2018). Iron transport kinetics through blood-brain barrier endothelial cells. Biochim. Biophys. Acta. Gen. Subj. 1862, 1168-1179. doi: 10.1016/j.bbagen.2018.02.010

Kim, H. W., Shin, J. A., Kim, H. J., Ahn, J. H., and Park, E. M. (2020). Enhanced repair processes and iron uptake by ischemic preconditioning in the brain during the recovery phase after ischemic stroke. Brain Res. 1750:147172. doi: 10.1016/j.brainres.2020.147172

Langkammer, C., Schweser, F., Krebs, N., Deistung, A., Goessler, W., Scheurer, E., et al. (2012). Quantitative susceptibility mapping (QSM) as a means to measure brain iron? a post mortem validation study. Neuroimage 62, 1593-1599. doi: 10.1016/j.neuroimage.2012.05.049

Lim, I. A., Faria, A. V., Li, X., Hsu, J. T., Airan, R. D., Mori, S., et al. (2013). Human brain atlas for automated region of interest selection in quantitative susceptibility mapping: application to determine iron content in deep gray matter structures. Neuroimage 82, 449-469. doi: 10.1016/j.neuroimage.2013.05.127

Linville, R. M., DeStefano, J. G., Sklar, M. B., Xu, Z., Farrell, A. M., Bogorad, M. I., et al. (2019). Human iPSC-derived blood-brain barrier microvessels: validation of barrier function and endothelial cell behavior. Biomaterials 190-191, 24-37. doi: 10.1016/j.biomaterials.2018.10.023

Liu, H., Chen, R., Wang, J., Chen, S., Xiong, C., Wang, J., et al. (2014). 1,5diaminonaphthalene hydrochloride assisted laser desorption/ionization mass spectrometry imaging of small molecules in tissues following focal cerebral ischemia. Anal. Chem. 86, 10114-10121. doi: 10.1021/ ac5034566
Masaldan, S., Bush, A. I., Devos, D., Rolland, A. S., and Moreau, C. (2019). Striking while the iron is hot: iron metabolism and ferroptosis in neurodegeneration. Free Radic. Biol. Med. 133, 221-233. doi: 10.1016/j.freeradbiomed.2018.09.033

McCarthy, R. C., and Kosman, D. J. (2015). Iron transport across the bloodbrain barrier: development, neurovascular regulation and cerebral amyloid angiopathy. Cell Mol. Life Sci. 72, 709-727. doi: 10.1007/s00018-014-1771-4

Mohan, K. M., Crichton, S. L., Grieve, A. P., Rudd, A. G., Wolfe, C. D., and Heuschmann, P. U. (2009). Frequency and predictors for the risk of stroke recurrence up to 10 years after stroke: the South London stroke register. J. Neurol. Neurosurg. Psychiatry 80, 1012-1018. doi: 10.1136/jnnp. 2008.170456

Munoz, P., and Humeres, A. (2012). Iron deficiency on neuronal function. Biometals 25, 825-835. doi: 10.1007/s10534-012-9550-x

Murakami, Y., Kakeda, S., Watanabe, K., Ueda, I., Ogasawara, A., Moriya, J., et al. (2015). Usefulness of quantitative susceptibility mapping for the diagnosis of Parkinson disease. AJNR Am. J. Neuroradiol. 36, 1102-1108. doi: 10.3174/ajnr.A4260

Oppo, K., Leen, E., Angerson, W. J., Cooke, T. G., and McArdle, C. S. (1998). Doppler perfusion index: an interobserver and intraobserver reproducibility study. Radiology 208, 453-457. doi: 10.1148/radiology.208.2.9680575

Pang, C., Cao, L., Wu, F., Wang, L., Wang, G., Yu, Y., et al. (2015). The effect of trans-resveratrol on post-stroke depression via regulation of hypothalamus-pituitary-adrenal axis. Neuropharmacology 97, 447-456. doi: 10.1016/j.neuropharm.2015.04.017

Petty, R. G., Bonner, D., Mouratoglou, V., and Silverman, M. (1996). Acute frontal lobe syndrome and dyscontrol associated with bilateral caudate nucleus infarctions. Br. J. Psychiatry 168, 237-240. doi: 10.1192/bjp. 168.2.237

Rathore, K. I., Redensek, A., and David, S. (2012). Iron homeostasis in astrocytes and microglia is differentially regulated by TNF-alpha and TGF-beta1. Glia 60, 738-750. doi: 10.1002/glia.22303

Rouault, T. A. (2001). Iron on the brain. Nat. Genet. 28, 299-300. doi: $10.1038 / 91036$

Schafer, A., Wharton, S., Gowland, P., and Bowtell, R. (2009). Using magnetic field simulation to study susceptibility-related phase contrast in gradient echo MRI. Neuroimage 48, 126-137. doi: 10.1016/j.neuroimage.2009.05.093

Shin, J. A., Kim, Y. A., Kim, H. W., Kim, H. S., Lee, K. E., Kang, J. L., et al. (2018). Iron released from reactive microglia by noggin improves myelin repair in the ischemic brain. Neuropharmacology 133, 202-215. doi: 10.1016/j.neuropharm.2018.01.038

Shmueli, K., de Zwart, J. A., van Gelderen, P., Li, T. Q., Dodd, S. J., and Duyn, J. H. (2009). Magnetic susceptibility mapping of brain tissue in vivo using MRI phase data. Magn. Reson. Med. 62, 1510-1522. doi: 10.1002/mrm.22135

Siesjo, B. K. (1988). Acidosis and ischemic brain damage. Neurochem. Pathol. 9, 31-88.

Simpson, I. A., Ponnuru, P., Klinger, M. E., Myers, R. L., Devraj, K., Coe, C. L., et al. (2015). A novel model for brain iron uptake: introducing the concept of regulation. J. Cereb. Blood Flow Metab. 35, 48-57. doi: 10.1038/jcbfm. 2014.168

Sun, H., Walsh, A. J., Lebel, R. M., Blevins, G., Catz, I., Lu, J. Q., et al. (2015). Validation of quantitative susceptibility mapping with perls' iron staining for subcortical gray matter. Neuroimage 105, 486-492. doi: 10.1016/j.neuroimage.2014.11.010

Thomas, G. E. C., Leyland, L. A., Schrag, A. E., Lees, A. J., Acosta-Cabronero, J., and Weil, R. S. (2020). Brain iron deposition is linked with cognitive severity in Parkinson's disease. J. Neurol. Neurosurg Psychiatry 91, 418-425. doi: 10.1136/jnnp-2019-322042

Wang, Q., Tang, X. N., and Yenari, M. A. (2007). The inflammatory response in stroke. J. Neuroimmunol. 184, 53-68. doi: 10.1016/j.jneuroim.2006.11.014

Ward, R. J., Zucca, F. A., Duyn, J. H., Crichton, R. R., and Zecca, L. (2014). The role of iron in brain ageing and neurodegenerative disorders. Lancet Neurol. 13, 1045-1060. doi: 10.1016/S1474-4422(14)70117-6

Yan, N., and Zhang, J. J. (2019). The emerging roles of ferroptosis in vascular cognitive impairment. Front. Neurosci. 13:811. doi: 10.3389/fnins. 2019.00811

Yushmanov, V. E., Kharlamov, A., Yanovski, B., LaVerde, G., Boada, F. E., and Jones, S. C. (2013). Correlated sodium and potassium imbalances within the ischemic core in experimental stroke: a $23 \mathrm{Na}$ MRI and 
histochemical imaging study. Brain Res. 1527, 199-208. doi: 10.1016/j.brainres. 2013.06.012

Zhang, X., Haaf, M., Todorich, B., Grosstephan, E., Schieremberg, H., Surguladze, N., et al. (2005). Cytokine toxicity to oligodendrocyte precursors is mediated by iron. Glia 52, 199-208. doi: 10.1002/glia.20235

Zhang, X., Surguladze, N., Slagle-Webb, B., Cozzi, A., and Connor, J. R. (2006). Cellular iron status influences the functional relationship between microglia and oligodendrocytes. Glia 54, 795-804. doi: 10.1002/ glia.20416

Zhang, X., Zhao, H. H., Li, D., and Li, H. P. (2019). Neuroprotective effects of matrix metalloproteinases in cerebral ischemic rats by promoting activation and migration of astrocytes and microglia. Brain Res. Bull. 146, 136-142. doi: 10.1016/j.brainresbull.2018.11.003

Zhang, Z. G., and Chopp, M. (2015). Promoting brain remodeling to aid in stroke recovery. Trends Mol. Med. 21, 543-548. doi: 10.1016/j.molmed.2015.07.005
Zhao, L. R., and Willing, A. (2018). Enhancing endogenous capacity to repair a stroke-damaged brain: An evolving field for stroke research. Prog. Neurobiol. 163-164, 5-26. doi: 10.1016/j.pneurobio.2018.01.004

Conflict of Interest: The authors declare that the research was conducted in the absence of any commercial or financial relationships that could be construed as a potential conflict of interest.

Copyright (C) 2021 Du, Zhao, Liu, Chen, Gao, Wang, Liu, Liu and Ma. This is an open-access article distributed under the terms of the Creative Commons Attribution License (CC BY). The use, distribution or reproduction in other forums is permitted, provided the original author(s) and the copyright owner(s) are credited and that the original publication in this journal is cited, in accordance with accepted academic practice. No use, distribution or reproduction is permitted which does not comply with these terms. 